\title{
Microbiome-Metabolomics Reveals Prebiotic Benefits of Fucoidan Supplementation in Mice
}

\author{
Jingyi Yuan ${ }^{1,2}$, Song Qin ${ }^{1,3}{ }^{\oplus}$, Wenjun $\mathrm{Li}^{1,3}$, Yubing Zhang ${ }^{1,2}{ }^{(D}$, Yuting Wang ${ }^{1}$, Xiulian Chang ${ }^{2}$ and Lili Li ${ }^{1,3, *}$ \\ 1 Yantai Institute of Coastal Zone Research, Chinese Academy of Sciences, Yantai 264003, China; \\ y1356232380@163.com (J.Y.); sqin@yic.ac.cn (S.Q.); wjli@yic.ac.cn (W.L.); \\ 202000370028@s.ytu.edu.cn (Y.Z.); wangyuting1820@163.com (Y.W.) \\ 2 College of Life Sciences, Yantai University, Yantai 264005, China; changxiulian7@126.com \\ 3 Center for Ocean Mega-Science, Chinese Academy of Sciences, Qingdao 266071, China \\ * Correspondence: 1lli@yic.ac.cn
}

check for updates

Citation: Yuan, J.; Qin, S.; Li, W.; Zhang, Y.; Wang, Y.; Chang, X.; Li, L. Microbiome-Metabolomics Reveals Prebiotic Benefits of Fucoidan Supplementation in Mice. J. Mar. Sci. Eng. 2021, 9, 505. https://doi.org/ 10.3390/jmse 9050505

Academic Editor: Azizur Rahman

Received: 9 April 2021

Accepted: 2 May 2021

Published: 8 May 2021

Publisher's Note: MDPI stays neutral with regard to jurisdictional claims in published maps and institutional affiliations.

Copyright: (C) 2021 by the authors. Licensee MDPI, Basel, Switzerland. This article is an open access article distributed under the terms and conditions of the Creative Commons Attribution (CC BY) license (https:/ / creativecommons.org/licenses/by/ $4.0 /)$.

\begin{abstract}
Fucoidan is a kind of polysaccharide with antitumor and antioxidant properties, which is mainly isolated from brown algae. Although there are many reports about the prebiotic effects of polysaccharides on hosts, there are few reports about the effects of fucoidan on blood biochemical indexes, intestinal microbiome, and metabolic function on healthy hosts. We applied $16 \mathrm{~S}$ rRNA gene amplicon sequencing and LC-MS/MS metabolomics to evaluate the changes in the gut microbiome and metabolite profiles of fucoidan treatment in mice over 10 weeks. Fucoidan treatment modulated lipid metabolism, including significantly decreasing serum triglyceride level in healthy mice. Fucoidan also significantly inhibited serum lipopolysaccharide-binding protein (LBP) concentration, a biomarker of endotoxemia. Correlation analysis further showed that Lactobacillus animalis populations that were enriched by fucoidan demonstrated significantly negative correlations with serum triglyceride level. The abundance of Lactobacillus gasseri and Lactobacillus reuteri, increased by fucoidan supplementation, demonstrated significantly negative correlation with lipopolysaccharide-binding protein levels. Lactobacillus gasseri also demonstrated significantly positive correlations with three tryptophan-related metabolites, including indoleacrylic acid, 3indoleacrylic acid, and 5-hydroxytryptamine, which were all increased by fucoidan administration. Combined with the previous evidence, the results indicate that fucoidan exerts prebiotic effects, such as lipid metabolism suppression and metabolic endotoxemia suppression, by modulating the abundance of gut microbiota, such as Lactobacillus animalis, Lactobacillus gasseri, and Lactobacillus reuteri, as well as microbiota-dependent metabolites, such as tryptophan-related metabolites.
\end{abstract}

Keywords: fucoidan; microbiome; metabolomics; gut microbiota

\section{Introduction}

The gut microbiota is a complex microbial ecosystem, the homeostasis of which is essential for host health. In the past decade, research has revealed the association or even the causal relationship between gut microbiota and many diseases such as intestinal inflammation, metabolic syndrome, and cancer. The gut microbiota also affects the host's metabolic phenotype through the production of extremely diverse metabolites. These metabolites penetrate into the blood circulation and interact with host cells, or become messengers of signal transmission and thus influence host phenotype. Metabolomics analysis increases the understanding of the function and specificity of the microbiome [1]. For example, the gut microbiota metabolizes tryptophan to produce indole and indole derivatives, which improve alcoholic liver disease and enhance host intestinal immunity [2,3].

Polysaccharides, such as fucoidan, pass through the upper gastrointestinal system intact and are fermented by gut microorganisms in the lower gastrointestinal system, the metabolites of which further influence immunity [4] and obesity risk [5]. The evidence of the relationship between polysaccharides, obesity, and other metabolic diseases has 
gradually revealed that the high-molecular-weight polysaccharide components in Cordyceps mycelium promote the growth of specific intestinal bacteria such as Parabacteroides goldsteinii, thereby improving obesity and related metabolic disorders in mice [6]. Inulin improves the intestinal microbes of obese mice and inhibits the increase in intestinal permeability caused by the Western diet [7,8]. In addition to fungi and terrestrial plants, seaweeds also contain a large number of bioactive polysaccharides.

Fucoidan is mainly composed of the L-fucose and sulfate group, which is mainly isolated from brown seaweeds such as Undaria pinnatifida. Fucoidan is extensively added in dietary and health foods because of its anti-oxidant and antitumor effects. Diet has emerged as a pivotal factor shaping the community structure and function of the gut microbiota, directly or indirectly affecting host metabolism, immunity, and intestinal barriers [9]. In recent years, dietary fiber, such as polysaccharides, was encouraged as a dietary additive by experts in medicine and nutrition. A number of studies revealed the beneficial effects of several polysaccharides, but little information was known about the effects of fucoidan on healthy hosts, especially its influence on biochemical indexes, intestinal microbiome, and metabolic function, as well as the relationship among them. A better understanding of the effects of fucoidan on the gut microbiome will help us to explain the prebiotic benefits of fucoidan. In this study, microbiome-metabolomics was used to reveal the health effects of fucoidan on mice.

\section{Materials}

\subsection{Animals and Diets}

SPF C57BL/6J mice (six weeks old, male) were purchased from Jinan Pengyue Experimental Animal Technology Co., Ltd. (Jinan, China). Mice were housed under environmentally controlled conditions (with humidity of $40-60 \%$, temperature of $20-25{ }^{\circ} \mathrm{C}$, and $12 \mathrm{~h} / 12 \mathrm{~h}$ light/dark cycles). All the mice had free access to water and corresponding intervention diets. After a week of adaptation to the breeding environment, mice were divided into two groups ( $n=8$ for each group): the control and fucoidan group (FU group). All mice were fed a normal chow diet (NCD, D12450B) for 10 weeks. Mice in the FU group drank water containing 1\% fucoidan (Qingdao Bright Moon Seaweed Group Co., Ltd., Qingdao, China) for 10 weeks. Body weight was monitored every two weeks. Feces were collected individually at the end of 10 weeks, and stored in liquid nitrogen immediately. Animal treatment was ratified by the Animal Protection Ethics Committee of Yantai University. Animal experiments were in line with the Declaration of Helsinki and international guidelines on animal experiments.

\subsection{Biochemical Analyses}

At the end of the 10th week, blood samples of approximately $1 \mathrm{~mL}$ were drawn from the orbit. Blood was centrifuged at $3000 \mathrm{rpm}$ for $15 \mathrm{~min}$ at $4{ }^{\circ} \mathrm{C}$; then, the serum was separated and collected for further analysis. Levels of serum triglyceride (TG) and total cholesterol (TC) were detected with an automatic biochemistry analyzer (Roche Diagnostics, Cobas c 311, Basel, Switzerland). Serum lipopolysaccharide binding protein (LBP) concentration was analyzed by the Mouse LBP ELISA Kit (Abcam, Cambridge, UK).

\subsection{Microbiota Analysis}

Microbiota samples were extracted from feces using the QIAamp DNA stool kit (QIAGEN Inc., Germantown, MD, USA). The variable V3-V4 regions of the $16 \mathrm{~S}$ rRNA genes were amplified by the primers of 515F 'GTGCCAGCMGCCGCGGTAA' and 806R 'GGACTACHVGGGTWTCTAAT'. The purified amplicons were analyzed using paired-end sequencing on an Illumina NovaSeq System (San Diego, CA, USA). The sequences were clustered into operational taxonomic units (OTUs) with $97 \%$ identity. The unweighted UniFrac distance matrix was used to perform ANOSIM statistical tests through QIIME (Version 1.9.1). The original raw sequence data of the mouse gut microbiota were deposited in NCBI Sequence Read Archive (SRA) under the accession number SUB9310543. 


\subsection{Metabolome Analysis}

Metabolome samples of mice feces were analyzed using Exion LC (SCIEX) equipped with a QTRAP6500+ mass spectrometer (SCIEX, Framingham, MA, USA). Samples were detected using a BEH C8 column $(1.7 \mu \mathrm{m} \times 2.1 \mathrm{~mm} \times 100 \mathrm{~mm}$, Waters $)$ with a flow rate of $0.35 \mathrm{~mL} / \mathrm{min}$ in positive electrospray ionization mode. The mobile phase was $0.1 \%(v / v)$ formic acid-water and $0.1 \%(v / v)$ formic acid-acetonitrile. Samples were detected using a HSS T3 column $(3.5 \mu \mathrm{m} \times 4.6 \mathrm{~mm} \times 250 \mathrm{~mm}$, Waters $)$ with a flow rate of $0.35 \mathrm{~mL} / \mathrm{min}$ in negative electrospray ionization mode. The mobile phase was $6.5 \mathrm{mmol} / \mathrm{L}$ ammonium bicarbonate and $6.5 \mathrm{mmol} / \mathrm{L}$ ammonium bicarbonate- $95 \%(v / v)$ methanol. Metabolites were further analyzed according to a previous method [10].

\subsection{Statistical Analysis}

Data were analyzed using GraphPad Prism version 6.0 (San Diego, CA, USA). The $t$-test was used to evaluate the difference between the two groups; $p<0.05$ was considered to indicate significantly different.

\section{Results}

\subsection{Effects of Fucoidan on Body Weight and Plasma Lipid Profile}

There was no significant difference in average body weight gain between the fucoidan and control groups (Figure A1). Fucoidan treatment after 10 weeks significantly decreased serum TG level and serum LBP concentration compared with the control group (Figure A1B,C). Compared with the control group, fucoidan supplementation reduced concentrations of total cholesterol (TC) and LBP by $13.62 \%$ and $43.61 \%$, respectively.

\subsection{Effects of Fucoidan on Microbial Communities}

The changes in bacterial communities after 10 weeks of fucoidan administration were analyzed by nonmetric multidimensional scaling, which demonstrated a distinct clustering of microbiota composition for the control and fucoidan groups (Figure A2). ANOSIM analysis further proved that fucoidan supplementation significantly modulated gut microbial communities (Figure A2B; $p=0.001$ ).

The two most numerous phyla were Firmicutes and Bacteroidetes, accounting for more than $97.44 \%$ of the total phyla (Figure A2C). The abundance of Firmicutes, Actinobacteria, and unidentified-bacteria phyla was significantly higher in the fucoidan group than in the control. However, fucoidan treatment significantly decreased the abundance of Bacteroidetes, Proteobacteria, Tenericutes, and Verrucomicrobia phyla (Figure A2C). Furthermore, we observed a marked increase in the abundance of the Lactobacillaceae family, and a significant decrease in the populations of Erysipelotrichaceae, Muribaculaceae, Ruminococcaceae, Bacteroidaceae, and Rikenellaceae families after fucoidan treatment (Figure A2D).

\subsection{Effects of Fucoidan on Bacterial Genera and Species}

Further analysis of microbial genera indicated that fucoidan treatment significantly reduced the abundance of Alistipes, Marvinbryantia, Dubosiella, unidentified Lachnospiraceae, Anaerotruncus, Lachnoclostridium, Parasutterella, Allobaculum, Faecalibaculum, Roseburia, Harryflintia, Intestinimonas, Parabacteroide, Muribaculum, Mucispirillum, Oscillibacter, Akkermansia, Tyzzerella, unidentified Ruminococcaceae, Bacteroides, and Ruminiclostridium genera, whereas fucoidan supplementation after 10 weeks significantly increased the abundance of Lactobacillus, Romboutsia, and Turicibacter genera (Figure A3). Moreover, Lactobacillus animalis, Lactobacillus gasseri, Lactobacillus reuteri, and Parabacteroides distasonis were significantly enriched in the fucoidan group (Figure A4A-D). However, a significant decrease in Bacteroide acidifaciens, Mucispirillum schaedleri, Alistipes finegoldii, and Clostridium leptum was observed after fucoidan treatment (Figure A4E-H). 


\subsection{Effects of Fucoidan on the Metabolomics}

Principal component analysis (PCA) demonstrated a distinct separation between the control and fucoidan groups (Figure A5A). All differential metabolites between the control and fucoidan groups are visualized in a volcano plot (Figure A5B). The larger variable importance in the projection (VIP) values were regarded as the more important biomarkers for differentiating the control and fucoidan groups. The most significant differential metabolic pathways were biosynthesis of amino acids, glycine, serine, and threonine metabolism; thiamine metabolism; and tryptophan metabolism (Figure A5C). Individual metabolites analysis further proved that tryptophan-related metabolites, including indoleacrylic acid, 3-indoleacrylic acid, and 5-hydroxytryptamine, were all significantly increased by fucoidan treatment (Figure A5D and Supplementary Figure S1). The levels of histidine and branched chain amino acids (BCAAs), including leucine, isoleucine and valine, were significantly increased by fucoidan supplementation (Figure A5D and Supplementary Figure S1).

\subsection{Spearman Correlation Analysis}

Correlation analysis showed that Lactobacillus animalis demonstrated significantly negative correlations with TG level (Figure A6), and the abundance of Parabacteroides distasonis, Bacteroides acidifaciens, Mucispirillum schaedleri, Alistipes finegoldii, and Clostridium leptum had significantly positive correlations with TG level (Figure A6). The abundance of Lactobacillus gasseri and Lactobacillus reuteri exhibited significantly negative correlations with LBP concentration (Figure A6). The abundance of Mucispirillum schaedleri exhibited significantly positive correlation with LBP concentration (Figure A6).

Tryptophan-related metabolites in mice feces, including methyl indole-3-acetate, 3indoleacrylic acid, and 5-hydroxytryptamine, showed significantly positive correlation with Lactobacillus gasseri populations (Figure A7A). Most of the differential organic acid and its derivatives, such as levels of 5-aminolevulinate, cis-4-hydroxy-D-proline, 6-aminocaproic acid, urocanic acid, indoleacrylic acid, $N$-methyl-a-aminoisobutyric acid, aminomalonic acid, guanidineacetic acid, DL-o-tyrosine, and L-homoserine, were noticeably increased in the fucoidan group and showed a positive correlation with Lactobacillus animalis and Lactobacillus gasseri populations (Figures A5D and A7A). Conversely, correlation analysis between mice gut microbiota species and amino acid in mice feces and its derivatives indicated that Lactobacillus animalis, Lactobacillus gasseri, and Lactobacillus reuteri populations demonstrated negative correlations with concentrations of L-methionine, DL-leucine, $\mathrm{N}$ acetyl-L-glutamic acid, 3-nitro tyrosine, D-phenylalanine, DL-threonine, and DL-tyrosine in mice feces (Figure A7B); and Parabacteroides distasonis, Bacteroides acidifaciens, Mucispirillum schaedleri, Alistipes finegoldii, and Clostridium leptum populations demonstrated positive correlations with L-phenylalanine, L-tyrosine, L-tryptophan, L-isoleucine, L-methionine, D-methionine, DL-leucine, $N$-acetyl-L-glutamic acid, 3-nitro-tyrosine, D-phenylalanine, DLthreonine, DL-tyrosine, L-methionine sulfone, asparagine, S-sulfo-L-cysteine, DL-norvaline, isoleucine, D-norleucine, DL-valine, norvaline, methionine, tyrosine, alanine- glycine, histidine, aspartate-phenylalanine, D(-)-arginine, DL-arginine, and D-histidine (Figure A7B).

\section{Discussion}

The beneficial effects of plant-derived polysaccharides on host health have gradually been revealed, for example, inulin alleviated endotoxemia and inflammation induced by high-fat diet via its prebiotic influence in C57BL/6J mice [11]. In our study, fucoidan supplementation decreased serum TG and TC levels in mice. Higher TG and TC levels represent a disorder in lipid metabolism; high TC and TG levels increase the risk of pancreatitis and cardiovascular diseases [12,13]. Furthermore, correlation analysis indicated that Lactobacillus gasseri and Lactobacillus animalis had negative correlations with TG level. These two species were also significantly enriched by fucoidan. Lactobacillus gasseri reduced body weight gain and improved glucose tolerance in rats via prompting energy expenditure [14]. Lactobacillus gasseri supplementation significantly decreased visceral fat weight and liver TG concentration in KK-Ay mice (animal model of type 2 diabetes) fed a high-fat diet [15]. 
It was indicated that the reduction in TG levels after fucoidan administration might be associated with the enrichment in Lactobacillus gasseri and Lactobacillus animalis populations.

Lipopolysaccharide (LPS)-binding protein acts as a marker of metabolic endotoxemia. Fucoidan administration demonstrated an ability to reduce serum LBP in mice in our results. Previous research has demonstrated that dysfunction of intestinal permeability or damaged epithelium leads to enhancing the LPS in the intestinal lumen, and LPS further entering the systemic circulation causes metabolic diseases and inflammation [16-18]. Intraperitoneal administration of LPS caused neuroinflammatory response and spatial learning impairments [19]. LPS led to testicular damage, apoptosis, and inflammation in spermatogonial stem cells [20]. Interestingly, Lactobacillus gasseri and Lactobacillus reuteri, enriched by fucoidan supplementation, showed negative associations with serum LPS concentration, which is consistent with the general anti-inflammatory characteristics of Lactobacillus gasseri and Lactobacillus reuteri populations [21,22].

Tryptophan generated from gut microorganisms is an important signaling molecule in the interaction between host and microorganisms, which might contribute to the enteroendocrine system and metabolic homeostasis including glucose metabolism [23]. A recent study reported an association between tryptophan-related metabolites and the activity of Aryl hydrocarbon receptor (AhR), suggesting that these metabolites might serve as biomarkers representative of intestinal AhR activity to contribute to mucosal homeostasis [24]. In our study, the concentrations of tryptophan-related metabolites indole acrylic acid, 3-indoleacrylic acid, and 5-hydroxytryptamine in mice feces significantly increased after fucoidan supplementation, and the three metabolite concentrations were positively correlated with Lactobacillus animalis and Lactobacillus gasseri populations. Lactobacillus reuteri was reported to improve constipation via modulating serum 5-hydroxytryptamine concentration in patients with functional constipation [25].

A previous study found that indole acrylic acid promoted function of the intestinal epithelial barrier and reduced the inflammatory response in mice by promoting goblet cell differentiation and mucus formation, possibly mediated by AhR activation [23]. Indole acrylic acid was also proven to be absorbed through the intestinal epithelium and to enter systemic circulation, which exerts antioxidant and anti-inflammatory activity [23]. Additionally, 5-hydroxytryptamine was shown to improve intestinal inflammation, such as dextran sulfate sodium-induced colitis in mice [26]. Both indole acrylic acid and 5hydroxytryptamine reduced pro-inflammatory cytokines in macrophages and inhibited the migration of cells toward a chemokine [24]. The study suggested that fucoidan supplementation might improve host disorders and inflammatory responses by enhancing tryptophan-related metabolites, which is also supported by the enrichment in the Lactobacillus genus in mice.

Histidine, an essential amino acid in childhood, plays an important role in host homeostasis. This study found that after fucoidan supplementation, the concentration of histidine in mice feces increased by $61.77 \%$ compared with the control group. Histidinerelated metabolites histidine dipeptides, such as carnosine, are considered scavengers of reactive carbonyl species (RCS), which exhibited a potential pathogenic role in obesity and related metabolic disorders [27]. Importantly, histidine was observed to be associated with the progression of malignant tumors. A histidine-rich diet enhanced the inhibitory efficiency of low-dose methotrexate on solid tumors and reduced methotrexate toxicity in mice [28]. Kanarek et al. [29] also reported the similar finding that a histidine-rich diet increased the inhibitory sensitivity of antitumor drugs in mice via consuming the tetrahydrofolate of cancer cells.

Branched chain amino acids (BCAAs), including leucine, isoleucine, and valine, play important roles in regulating energy homeostasis, nutritional metabolism, intestinal health, and the immune response in both animal and human subjects [30]. In our results, fucoidan supplementation significantly increased the contents of leucine, isoleucine, and valine. Previous studies showed that leucine- and valine-related products 3-hydroxyisobutyrate and $\beta$-amino-isobutyric acid play signaling molecule roles, such as activating protein 
synthesis and insulin secretion [31]. Intake of BCAAs (leucine, isoleucine, and valine) contributed to the recovery of muscle after exercise and improvement in muscle function in human subjects. [32]. Leucine supplementation stimulated muscle protein synthesis by activating the mTOR signaling pathway [33].

In conclusion, fucoidan exerts prebiotic effects on healthy mice by lipid modulation and endotoxemia reduction, which might be associated with its capability to regulate the gut microbiota of species such as Lactobacillus animalis, Lactobacillus gasseri, and Lactobacillus reuteri, as well as its ability to modulate metabolomics, such as tryptophan-related metabolites (Figure A8). Our findings provide a dietary strategy to improve host metabolism.

Supplementary Materials: The following are available online at https:/ /www.mdpi.com/article/10 .3390 /jmse9050505/s1, Figure S1: Fucoidan treatment selectively promoted metabolites levels.

Author Contributions: L.L., X.C. and S.Q. designed the research. J.Y., Y.Z. and Y.W. were responsible for execution of the study, data collection, and analysis. L.L., S.Q., W.L. and X.C. interpreted the data and played major roles in drafting, writing, and revising this manuscript. All authors have read and agreed to the published version of the manuscript.

Funding: This research was funded by Youth Innovation Promotion Association of the Chinese Academy of Sciences, grant number 2018246, and Science and Technology Program of Yantai, grant number 2020MSGY076.

Institutional Review Board Statement: The study was conducted according to the guidelines of the Declaration of Helsinki, and approved by the Animal Ethics Association of Yantai University (protocol code YT2019-p-06, 26 March 2019).

Informed Consent Statement: Not applicable.

Data Availability Statement: Date will be made available from the corresponding author upon reasonable request.

Conflicts of Interest: The authors declare that they have no conflict of interest.

\section{Appendix A}

A

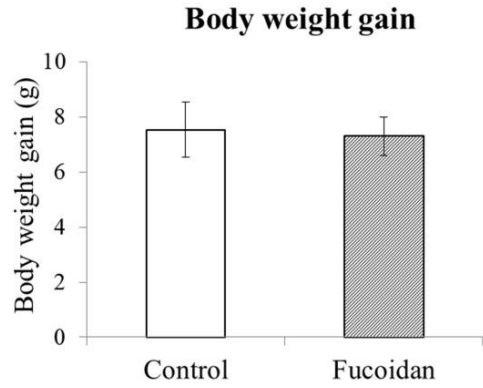

$\mathrm{C}$

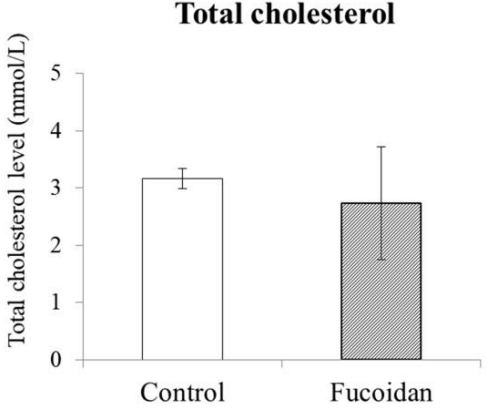

B

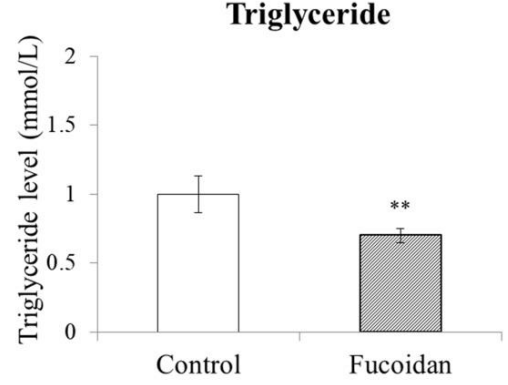

D

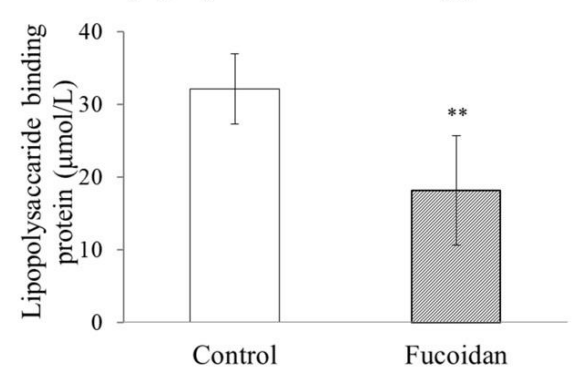

Figure A1. Effects of fucoidan supplementation in mice after 10 weeks on (A) body weight, (B) triglyceride, (C) total cholesterol, and (D) lipopolysaccharide-binding protein levels. ${ }^{* *} p<0.01$. Blank square, control group; grey square, fucoidan group. 
A

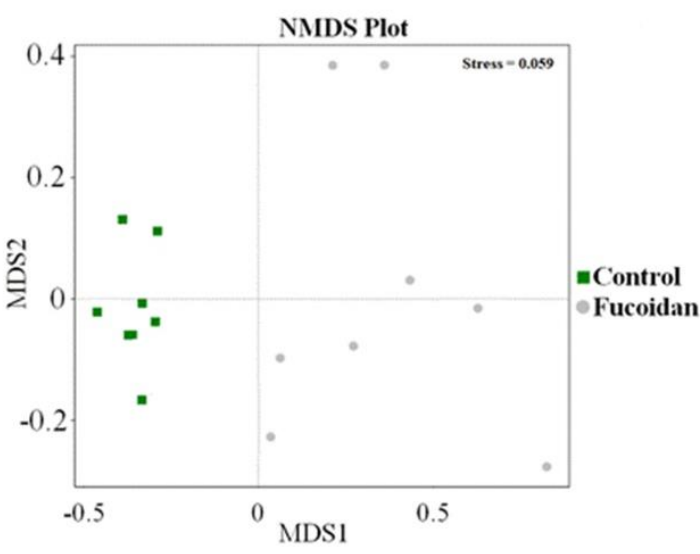

$\mathrm{C}$

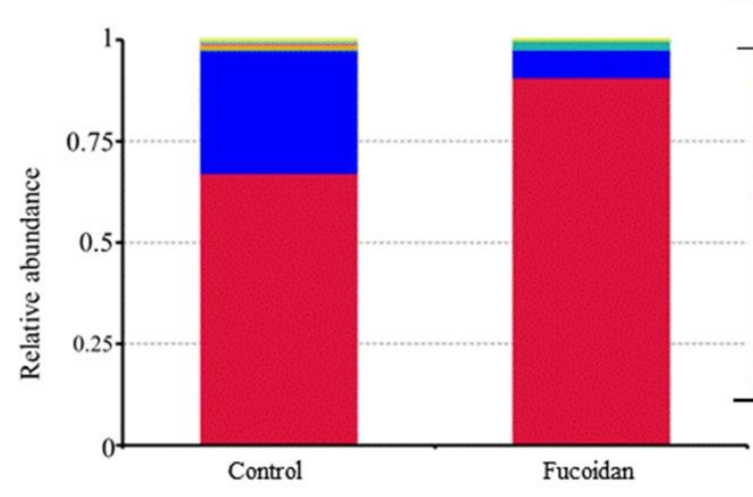

B

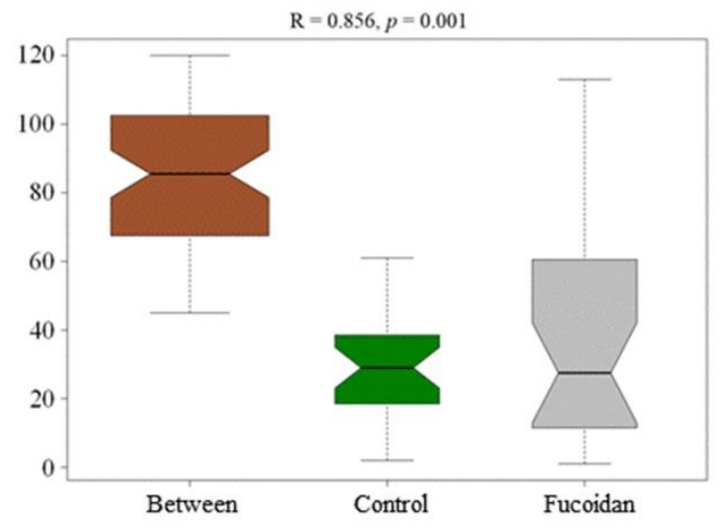

$\mathrm{D}$

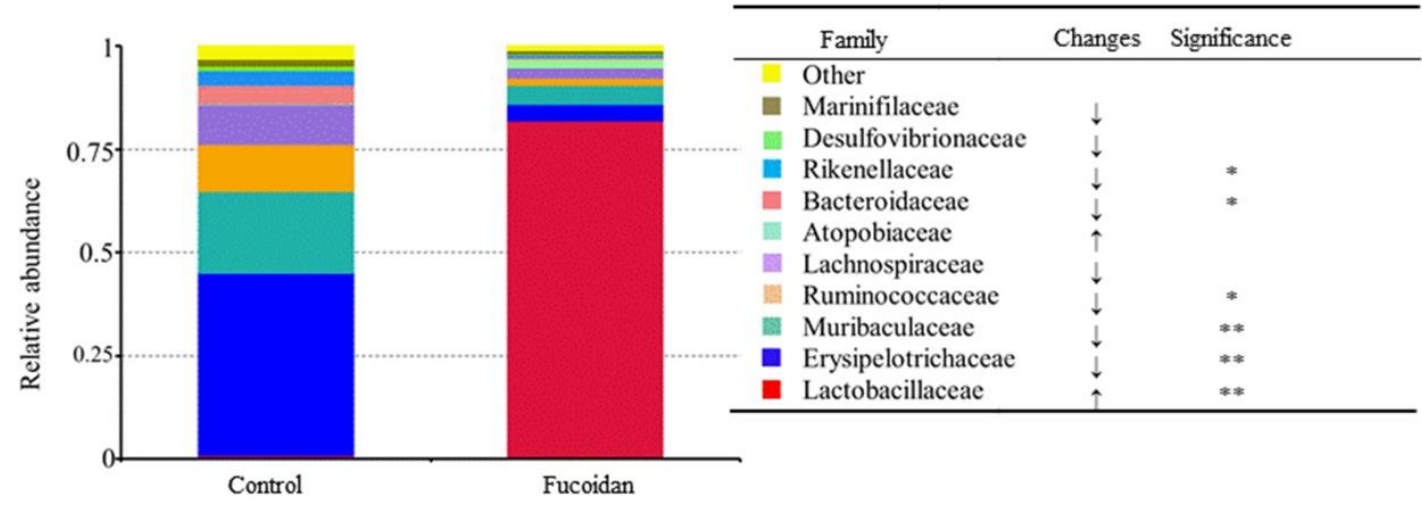

Figure A2. Bacterial community analysis. (A) Nonmetric multidimensional scaling (NMDS) and (B) ANOSIM analysis. The top 10 (C) phyla and (D) families. $\uparrow$, the genera increased by fucoidan supplementation in mice after 10 weeks; $\downarrow$, the genera decreased by fucoidan supplementation in mice after 10 weeks. ${ }^{*} p<0.05,{ }^{* *} p<0.01$. 


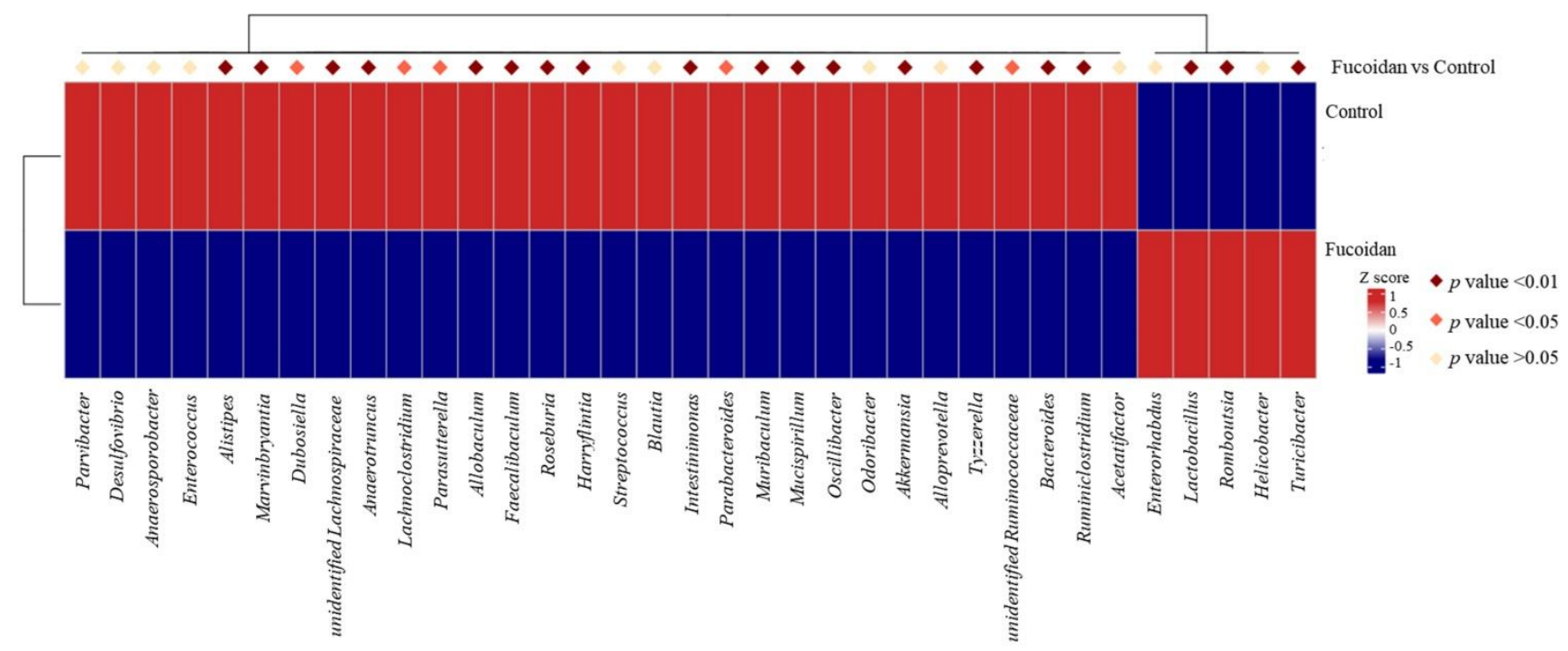

Figure A3. Fucoidan supplementation caused distinct shifts in the bacterial genera in mice.
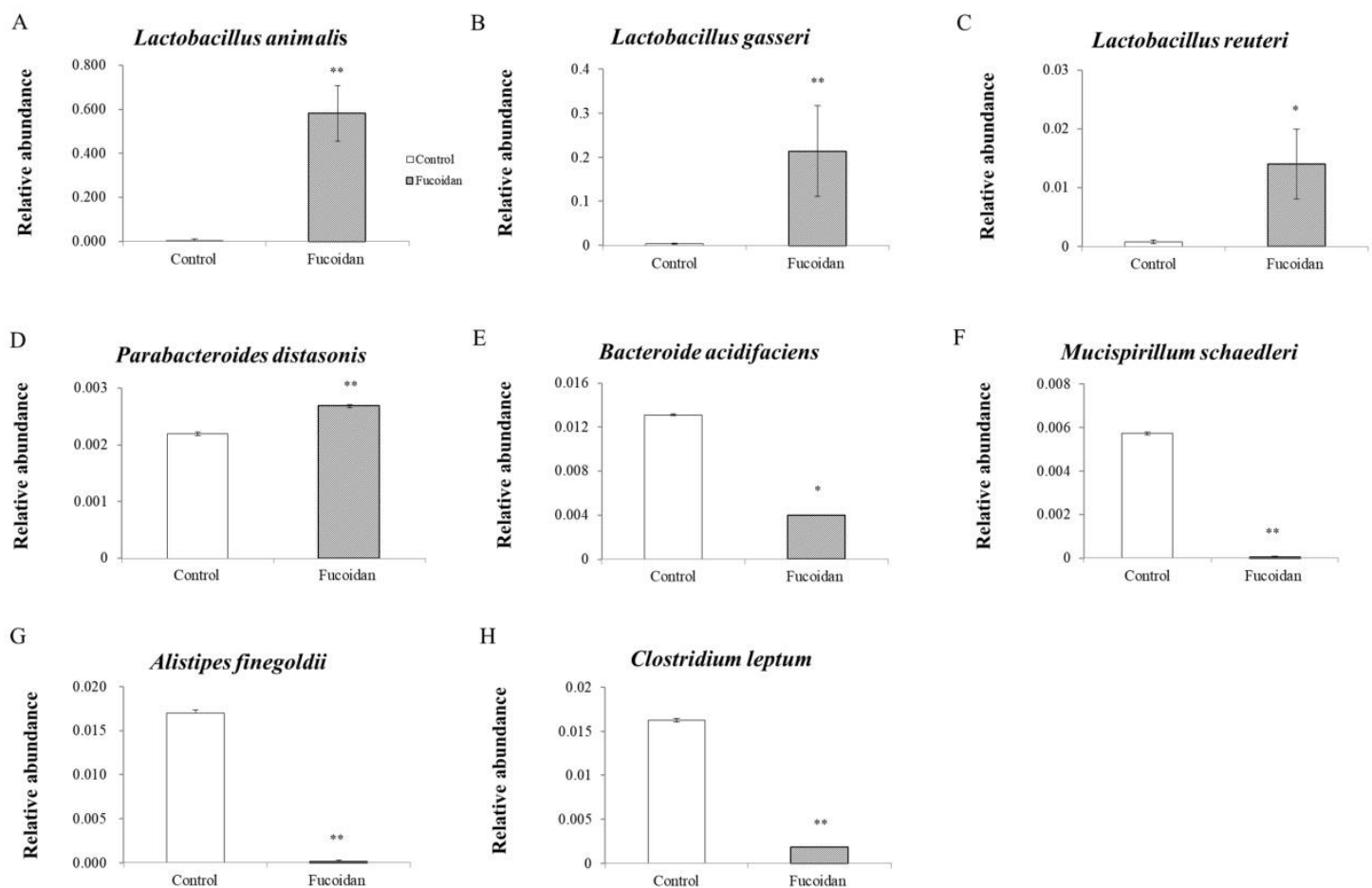

Figure A4. Fucoidan supplementation caused distinct shifts in the $(\mathbf{A}-\mathbf{H})$ bacterial species in mice. ${ }^{*} p<0.05,{ }^{* *} p<0.01$. Blank square, control group; grey square, fucoidan group. 
A
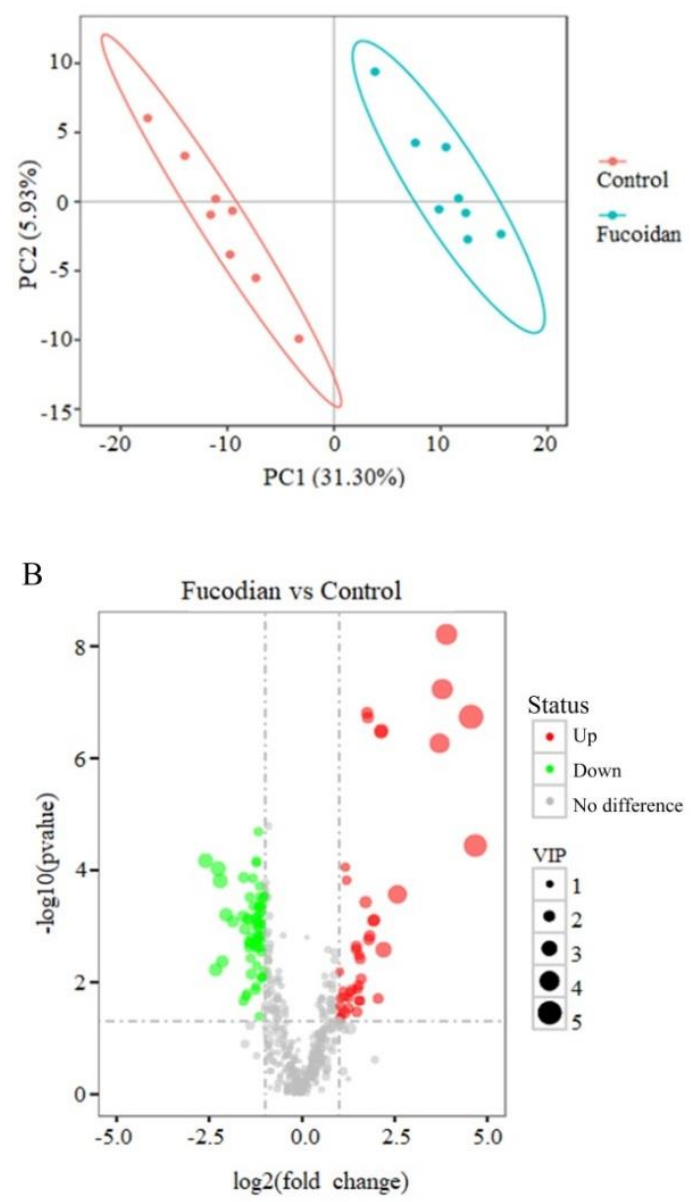

C

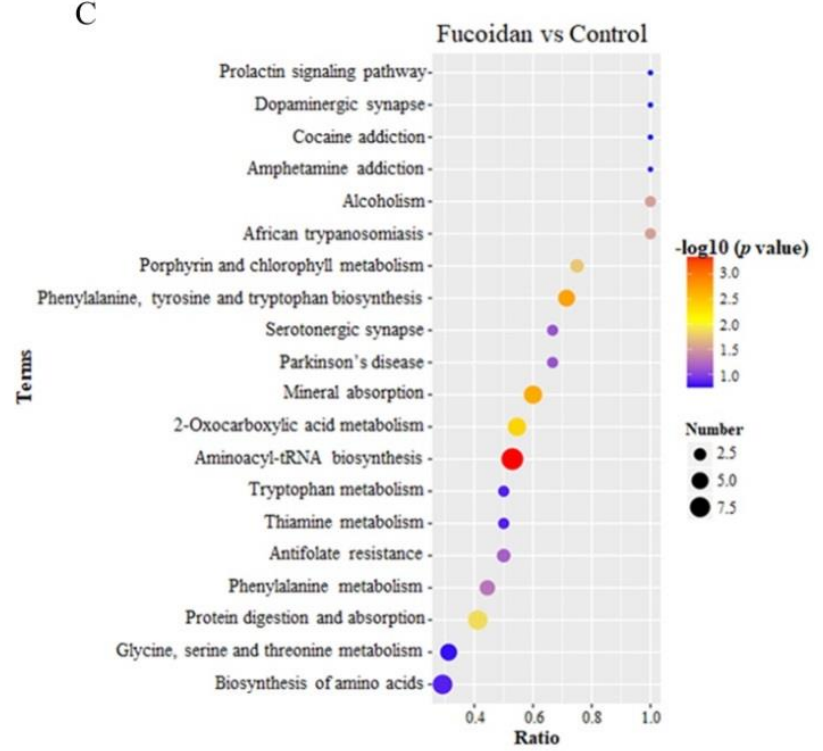

D

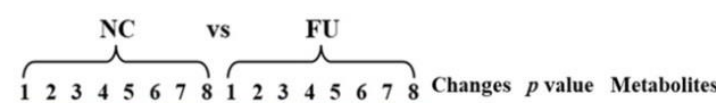

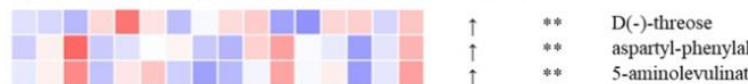

** DL-leucine

** a-cadinene

** N-acetyl-L-glutamic acid

** cis-4-hydroxy-D-proline

** 6-aminocaproic acid

** $\quad \beta$-cubebene

$\begin{array}{ll}* * & \beta \text {-cubebene } \\ * * & \text { methyl indole-3-acetate }\end{array}$

** 3-itnro-tyrosine

** D-phenylalanine

** L-phenylalanine

** DL-threonine

** farnesene

** DL-tyrosine

** urocanic acid

**. L-tyrosine

** L-methionine sulfone

** asparagine

** proline

* indoleacrylic acic

** 3-indoleacrylic acid

** DL-homoserine

** N-etmhyl-a-aminoisobutyric acid

** L-tryptophan

** aminomalonic acid

*** DL-proline

** S-sulfo-L-cystein

** DL-norvaline

** L-isoleucine

** D-pyrrolidine-2-carboxylic acid

** isoleucine

** guanidineacetic acid

** porphobilinogen

* porphobilinoge

* D- Dorlorcine

** N-methyl-L-arginine hydroc

** homoserin

** DL-o-tyrosine

** L-methionine

** DL-absorptionaline

** L-homoserine

norvaline

** D-fructose 6-pho

** tryptophan

** D-mannose 6-phospha

** methionine

** tyrosine

** trimethylamine $\mathrm{N}$-oxide

** L-alanyl-glycine

** histidine

"* $\gamma$-glutamylglutamic acid

** D-glucose 6-phosphate

** L-aspartyl-L-phenylalanine

** allothreonine

* 1,5-diaminopentane

** D-lactic acid

** D-methionine

** D(-)-arginine

** DL-arginin

** DL-arginine

"* D-histidine

** lysophospl

** glutamate

** DL-glutamic acid

** oleoyl-L-a-lysophosphatidic acid

- creatinine

hydroxypyruvic acid

levodopa

L-alanyl-L-leucine

5-hydroxytryptamine

Figure A5. Analysis of fecal metabolites in mice $(n=8)$. (A) Principal component analysis (PCA) and (B) volcano plot. Each dot represents one metabolite. Red dots represent the metabolites significantly upregulated and green dots represent the metabolites significantly downregulated by fucoidan treatment. The dot size represents the VIP value. (C) Metabolic pathways disturbed by fucoidan supplementation. The smaller the $p$ value, the more reliable and statistically significant the test. (D) All the differential metabolites between the control and fucoidan groups. $\uparrow$, upregulated by fucoidan administration after 10 weeks; $\downarrow$, downregulated by fucoidan administration after 10 weeks. ${ }^{*} p<0.05,{ }^{* *} p<0.01$. 


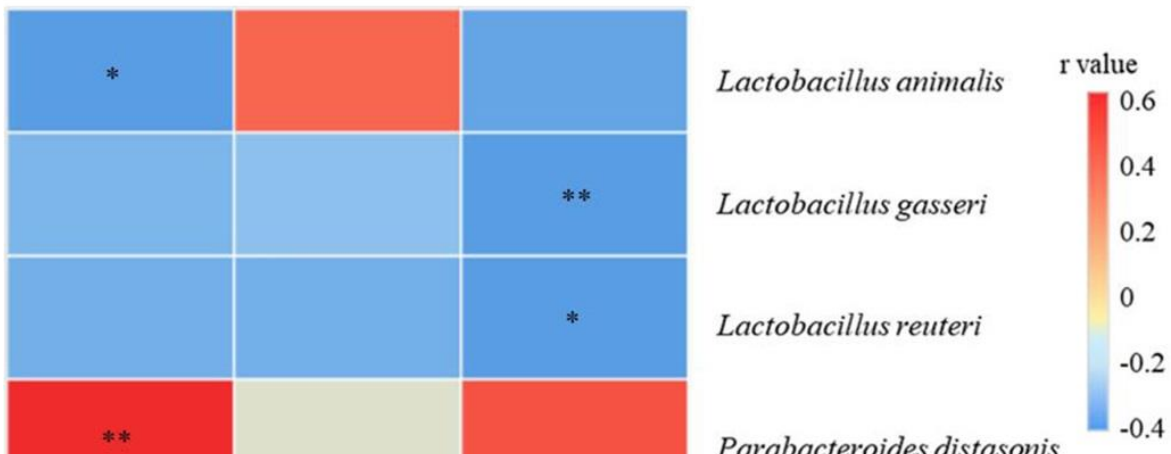

Parabacteroides distasonis

value

0.6

0.2

$-0.2$

$-0.4$

Bacteroides acidifaciens

Mucispirillum schaedleri

Alistipes finegoldii

Clostridium leptum

LBP

Figure A6. Spearman correlation analysis between bacterial species and serum biochemical indicators of TG, TC, and LBP in mice. TG, triglyceride. TC, total cholesterol. LBP, lipopolysaccharide-binding protein. $n=8$ for each group. The $\mathrm{r}$ value represents the correlation coefficient. ${ }^{*} p<0.05,{ }^{* *} p<0.01$.

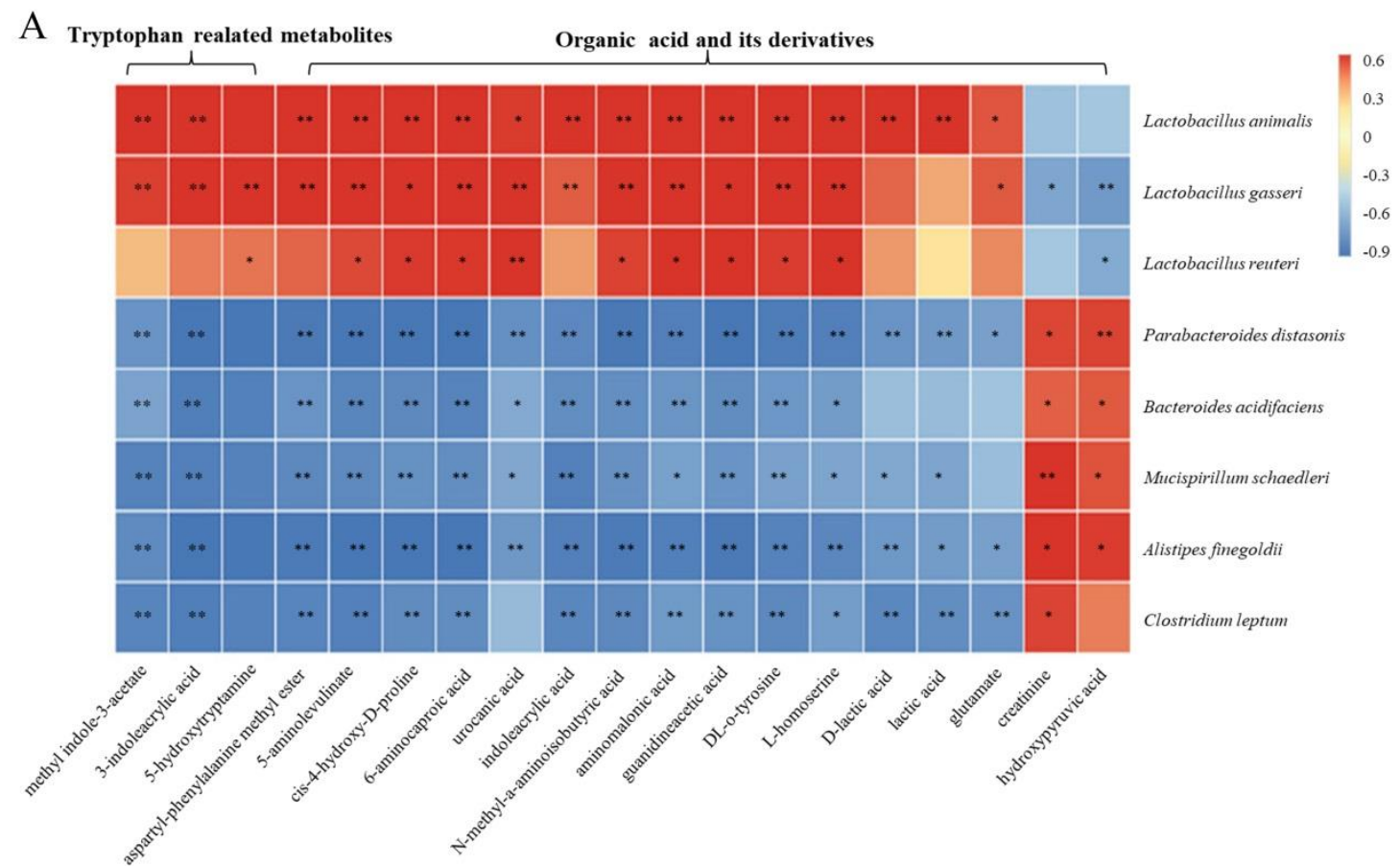

Figure A7. Cont. 


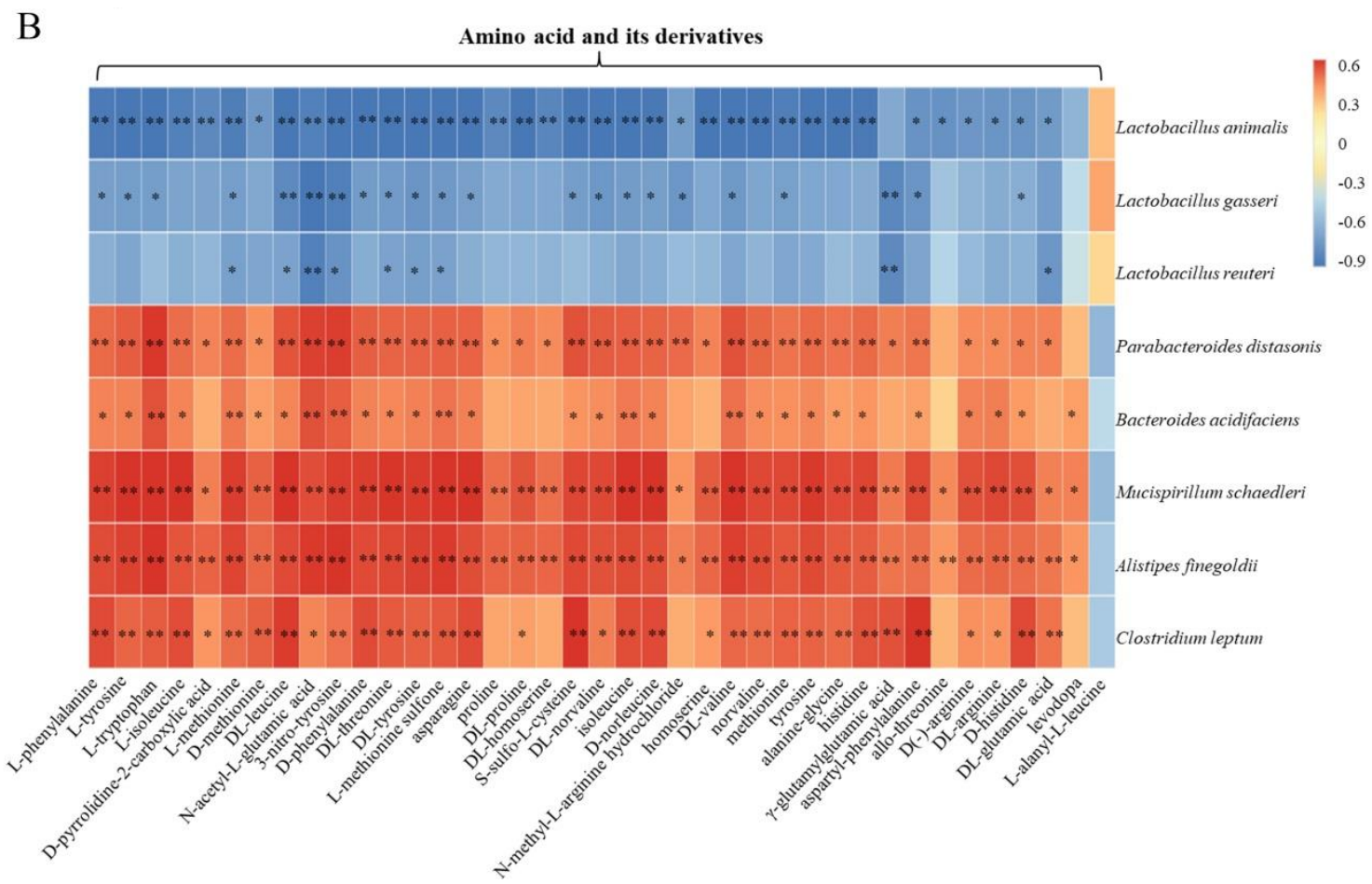

Figure A7. Spearman correlation analysis. (A) Correlation analysis between bacterial species and tryptophan-related metabolites, and organic acid and its derivatives. (B) Correlation analysis between bacterial species and amino acid and its derivatives. $n=8$ for each group. The $\mathrm{r}$ value represents the correlation coefficient. ${ }^{*} p<0.05,{ }^{* *} p<0.01$.

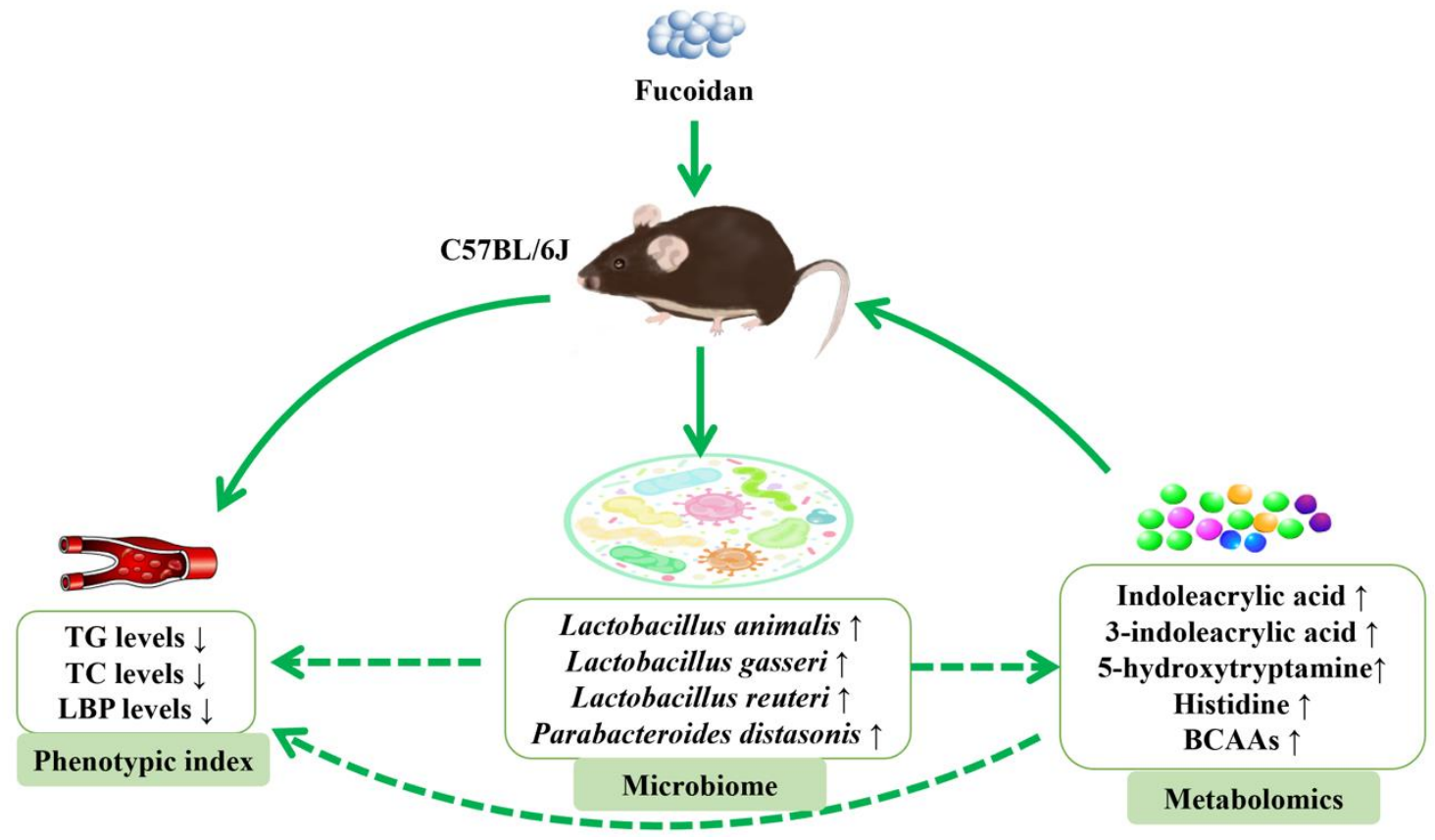

Figure A8. Microbiome-metabolomics reveals prebiotic benefits of fucoidan in mice. TG, triglyceride. TC, total cholesterol. LBP, lipopolysaccharide-binding protein. BCAAs, branched chain amino acids.

\section{References}

1. Hendrikx, T.; Schnabl, B. Indoles: Metabolites produced by intestinal bacteria capable of controlling liver disease manifestation. J. Intern. Med. 2019, 286, 32-40. [CrossRef] [PubMed]

2. Gao, J.; Xu, K.; Liu, H.; Liu, G.; Bai, M.; Peng, C.; Li, T.; Yin, Y. Impact of the gut microbiota on intestinal immunity mediated by tryptophan metabolism. Front. Cell. Infect. Microbiol. 2018, 8, 13. [CrossRef] [PubMed] 
3. Scott, S.A.; Fu, J.; Chang, P.V. Microbial tryptophan metabolites regulate gut barrier function via the aryl hydrocarbon receptor. Proc. Natl. Acad. Sci. USA 2020, 117, 19376-19387. [CrossRef] [PubMed]

4. Thomson, P.; Medina, D.A.; Ortuzar, V.; Gotteland, M.; Garrido, D. Anti-inflammatory effect of microbial consortia during the utilization of dietary polysaccharides. Food Res. Int. 2018, 109, 14-23. [CrossRef] [PubMed]

5. Chambers, E.S.; Byrne, C.S.; Morrison, D.J.; Murphy, K.G.; Preston, T.; Tedford, C.; Garcia-Perez, I.; Fountana, S.; SerranoContreras, J.I.; Holmes, E.; et al. Dietary supplementation with inulin-propionate ester or inulin improves insulin sensitivity in adults with overweight and obesity with distinct effects on the gut microbiota, plasma metabolome and systemic inflammatory responses: A randomised cross-over trial. Gut 2019, 68, 1430-1438. [CrossRef] [PubMed]

6. Wu, T.R.; Lin, C.S.; Chang, C.J.; Lin, T.L.; Martel, J.; Ko, Y.F.; Ojcius, D.M.; Lu, C.C.; Young, J.D.; Lai, H.C. Gut commensal Parabacteroides goldsteinii plays a predominant role in the anti-obesity effects of polysaccharides isolated from Hirsutella sinensis. Gut 2018, 68, 248-262. [CrossRef] [PubMed]

7. Schroeder, B.O.; Birchenough, G.M.H.; Stahlman, M.; Arike, L.; Johansson, M.E.V.; Hansson, G.C.; Backhed, F. Bifidobacteria or fiber protects against diet-induced microbiota-mediated colonic mucus deterioration. Cell Host Microbe 2018, 23, 27-40 e27. [CrossRef] [PubMed]

8. Zou, J.; Chassaing, B.; Singh, V.; Pellizzon, M.; Ricci, M.; Fythe, M.D.; Kumar, M.V.; Gewirtz, A.T. Fiber-mediated nourishment of gut microbiota protects against diet-induced obesity by restoring IL-22-mediated colonic health. Cell Host Microbe 2018, 23, 41-53 e44. [CrossRef] [PubMed]

9. Zmora, N.; Suez, J.; Elinav, E. You are what you eat: Diet, health and the gut microbiota. Nat. Rev. Gastroenterol. Hepatol. 2019, 16, 35-56. [CrossRef] [PubMed]

10. Wu, H.; Xie, S.; Miao, J.; Li, Y.; Wang, Z.; Wang, M.; Yu, Q. Lactobacillus reuteri maintains intestinal epithelial regeneration and repairs damaged intestinal mucosa. Gut Microbes 2020, 11, 997-1014. [CrossRef]

11. Li, L.L.; Wang, Y.T.; Zhu, L.M.; Liu, Z.Y.; Ye, C.Q.; Qin, S. Inulin with different degrees of polymerization protects against diet-induced endotoxemia and inflammation in association with gut microbiota regulation in mice. Sci. Rep. 2020, 10, 978. [CrossRef] [PubMed]

12. Piko, P.; Fiatal, S.; Werissa, N.A.; Bekele, B.B.; Racz, G.; Kosa, Z.; Sandor, J.; Adany, R. The effect of haplotypes in the cetp and lipc genes on the triglycerides to HDL-c ratio and its components in the roma and hungarian general populations. Genes 2020, 11, 56. [CrossRef] [PubMed]

13. Roubille, F.; Sultan, A.; Huet, F.; Leclercq, F.; Macia, J.C.; Gervasoni, R.; Delseny, D.; Akodad, M.; Roubille, C. Is hypertriglyceridemia atherogenic? Presse Med. 2018, 47, 757-763. [CrossRef] [PubMed]

14. Kim, J.; Yun, J.M.; Kim, M.K.; Kwon, O.; Cho, B. Lactobacillus gasseri BNR17 supplementation reduces the visceral fat accumulation and waist circumference in obese adults: A randomized, double-blind, placebo-controlled Trial. J. Med. Food 2018, 21, 454-461. [CrossRef]

15. Yonejima, Y.; Ushida, K.; Mori, Y. Lactobacillus gasseri NT decreased visceral fat through enhancement of lipid excretion in feces of KK-A(y) mice. Biosci. Biotechnol. Biochem. 2013, 77, 2312-2315. [CrossRef]

16. Citronberg, J.S.; Curtis, K.R.; White, E.; Newcomb, P.A.; Newton, K.; Atkinson, C.; Song, X.; Lampe, J.W.; Hullar, M.A. Association of gut microbial communities with plasma lipopolysaccharide-binding protein (LBP) in premenopausal women. ISME J. 2018, 12, 1631-1641. [CrossRef] [PubMed]

17. Pastor Rojo, O.; Lopez San Roman, A.; Albeniz Arbizu, E.; de la Hera Martinez, A.; Ripoll Sevillano, E.; Albillos Martinez, A. Serum lipopolysaccharide-binding protein in endotoxemic patients with inflammatory bowel disease. Inflamm. Bowel Dis. 2007, 13, 269-277. [CrossRef] [PubMed]

18. Roager, H.M.; Licht, T.R. Microbial tryptophan catabolites in health and disease. Nat. Commun. 2018, 9, 3294. [CrossRef] [PubMed]

19. Li, Y.; Liu, T.; Li, Y.; Han, D.; Hong, J.; Yang, N.; He, J.; Peng, R.; Mi, X.; Kuang, C.; et al. Baicalin ameliorates cognitive impairment and protects microglia from LPS-induced neuroinflammation via the SIRT1/HMGB1 pathway. Oxidative Med. Cell. Longev. 2020, 2020, 4751349. [CrossRef] [PubMed]

20. Yang, D.; Wei, Y.; Lu, Q.; Qin, D.; Zhang, M.; Du, X.; Xu, W.; Yu, X.; He, C.; Li, N.; et al. Melatonin alleviates LPS-induced endoplasmic reticulum stress and inflammation in spermatogonial stem cells. J. Cell. Physiol. 2020. [CrossRef]

21. Gebremariam, H.G.; Qazi, K.R.; Somiah, T.; Pathak, S.K.; Sjolinder, H.; Sverremark Ekstrom, E.; Jonsson, A.B. Lactobacillus gasseri suppresses the production of proinflammatory cytokines in -infected macrophages by inhibiting the expression of ADAM17. Front. Immunol. 2019, 10, 2326. [CrossRef]

22. Juarez, G.E.; Villena, J.; Salva, S.; de Valdez, G.F.; Rodriguez, A.V. Lactobacillus reuteri CRL1101 beneficially modulate lipopolysaccharide-mediated inflammatory response in a mouse model of endotoxic shock. J. Funct. Foods 2013, 5, $1761-1773$. [CrossRef]

23. Wlodarska, M.; Luo, C.; Kolde, R.; d'Hennezel, E.; Annand, J.W.; Heim, C.E.; Krastel, P.; Schmitt, E.K.; Omar, A.S.; Creasey, E.A.; et al. Indoleacrylic Acid Produced by Commensal Peptostreptococcus Species Suppresses Inflammation. Cell Host Microbe 2017, 22, 25-37 e26. [CrossRef]

24. Krishnan, S.; Ding, Y.; Saedi, N.; Choi, M.; Sridharan, G.V.; Sherr, D.H.; Yarmush, M.L.; Alaniz, R.C.; Jayaraman, A.; Lee, K. Gut microbiota-derived tryptophan metabolites modulate inflammatory response in hepatocytes and macrophages. Cell Rep. 2018, 23, 1099-1111. [CrossRef] [PubMed] 
25. Riezzo, G.; Chimienti, G.; Orlando, A.; D'Attoma, B.; Clemente, C.; Russo, F. Effects of long-term administration of Lactobacillus reuteri DSM-17938 on circulating levels of 5-HT and BDNF in adults with functional constipation. Benef. Microbes 2019, 10, 137-147. [CrossRef]

26. Kwon, Y.H.; Wang, H.; Denou, E.; Ghia, J.E.; Rossi, L.; Fontes, M.E.; Bernier, S.P.; Shajib, M.S.; Banskota, S.; Collins, S.M.; et al. Modulation of gut microbiota composition by serotonin signaling influences intestinal immune response and susceptibility to colitis. Cell. Mol. Gastroenterol. Hepatol. 2019, 7, 709-728. [CrossRef] [PubMed]

27. Anderson, E.J.; Vistoli, G.; Katunga, L.A.; Funai, K.; Regazzoni, L.; Monroe, T.B.; Gilardoni, E.; Cannizzaro, L.; Colzani, M.; De Maddis, D.; et al. A carnosine analog mitigates metabolic disorders of obesity by reducing carbonyl stress. J. Clin. Investig. 2018, 128, 5280-5293. [CrossRef] [PubMed]

28. Christian, F. Histidine metabolism boosts cancer therapy. Nature 2019, 559, 484-485.

29. Kanarek, N.; Petrova, B.; Sabatini, D.M. Dietary modifications for enhanced cancer therapy. Nature 2020, 579, 507-517. [CrossRef] [PubMed]

30. Ten Have, G.A.M.; Jansen, L.; Schooneman, M.G.; Engelen, M.; Deutz, N.E.P. Metabolic flux analysis of Branched-chain amino and keto acids (BCAA, BCKA) and beta-Hydroxy beta-methylbutyric acid (HMB) across multiple organs in the pig. Am. J. Physiol. Endocrinol. Metab. 2021. [CrossRef] [PubMed]

31. Neinast, M.; Murashige, D.; Arany, Z. Branched chain amino acids. Annu. Rev. Physiol. 2019, 81, 139-164. [CrossRef] [PubMed]

32. Arroyo-Cerezo, A.; Cerrillo, I.; Ortega, A.; FernAndez-PachOn, M.S. Intake of branched chain amino acids favors post-exercise muscle recovery and may improve muscle function: Optimal dosage regimens and consumption conditions. J. Sports Med. Phys. Fitness 2021. [CrossRef]

33. Wyant, G.A.; Abu-Remaileh, M.; Wolfson, R.L.; Chen, W.W.; Freinkman, E.; Danai, L.V.; Vander Heiden, M.G.; Sabatini, D.M mTORC1 activator SLC38A9 is required to efflux essential amino acids from lysosomes and use protein as a nutrient. Cell 2017, 171, 642-654.e612. [CrossRef] [PubMed] 